\title{
Long Term Outcomes of Iliac Artery Aneurysm (IAA) and Abdominal Aortic Aneurysm (AAA) Repair with Endovascular Procedures at the Parkview Heart Institute
}

\author{
Douglas Gray, M.D. ${ }^{1}$, Christopher Herrera, B.S. ${ }^{2}$, Kim Recht, N.P. ${ }^{1}$ \\ ${ }^{1}$ Parkview Heart Institute, ${ }^{2}$ Indiana University School of Medicine
}

Background: Favorable characteristics of the minimally invasive endovascular aneurysm repair (EVAR), such as a reduced recovery time, have led physicians and patients alike to elect for this procedure over open repair. Longitudinal study on endovascular repair has raised concerns regarding an increased rate of late failure leading to rupture and overall higher rates of re-intervention for patients.

Objective: This project compares the outcomes of endovascular stent graft repairs of AAA and IAA at Parkview Heart Institute over a certain time span.

Methods \& Design: This study is a retrospective review of patients $(\mathrm{N}=89)$ with AAA/IAA repair since 2014. The EPIC online medical record is the main tool in data extraction while statistical analysis was conducted via Microsoft Excel $(p=$ 0.05).

Results: Males, ages 51-75 y.o., White population, non-Hispanics, and BMI rating of 1-30 were observed to have more AAAs. A significant decrease in length of stay over historical averages was observed with at most 1-3 days and minimal complications. Most causes for re-interventions were due to endo-leaks. More than $60 \%$ of patients with AAA and EVAR have a significant past medical history of smoking, hypertension, and chronic obstructive pulmonary disease. Finally, most pre- and post- operative aneurysms sizes are within 41-60 mm in diameter.

Conclusion and Potential Impact: This study analyzed 89 patients that experienced AAA instead of IAA. Certain populations, such as being male, ages 51-75 y.0., White population, non-Hispanics, and BMl rating of 1-30 with past medical history characteristics are frequent in patients with EVAR. The procedure has minimal complications in comparison to open abdominal surgery, and this study will add to the literature by aiding physicians' determination of the best course of action for treating patients with AAA/IAA. 